\title{
bT-Locally Closed Sets and bT-Locally Continuous Functions In Supra Topological Spaces.
}

\author{
K.Krishnaveni And M.Vigneshwaran \\ Department of Mathematics Kongunadu Arts and Science College Coimbatore,Tamil Nadu, India.
}

\begin{abstract}
The aim of this paper is to introduce a decompositions namely supra bT-locally closed sets and define supra bT-locally continuous functions. This paper also discussed some of their properties.

Keyword S-BTLC set, S-BTL-continuous,S-BTL-irresolute.

\section{Introduction}

In 1983 Mashhour et al [8] introduced Supra topological spaces and studied S- continuous maps and $\mathrm{S}^{*}$ - continuous maps. In 2011,Bharathi.S[3] introduced and investigated several properties of generalization of locally b- closed sets. In 1997, Arokiarani .I[2] introduced and investigated some properties of regular generalized locally closed sets and RGL-continuous functions. In this paper, we define a new set called supra bT- locally closed and also define supra bT- locally continuous functions and investigated some of the basic properties for this class of functions.
\end{abstract}

\section{Preliminaries}

Definition 2.1[8,10] A subfamily of $\mu$ of $X$ is said to be a supra topology on $X$, if

(i) $\mathrm{X}, \phi \in \mu$

(ii) if $A_{i} \in \mu$ for all $i \in J$ then $\cup A_{i} \in \mu$.

The pair $(\mathrm{X}, \mu)$ is called supra topological space. The elements of $\mu$ are called supra open sets in $(\mathrm{X}, \mu)$ and complement of a supra open set is called a supra closed set.

Definition 2.2[10] (i) The supra closure of a set $A$ is denoted by $\mathrm{cl}^{\mu}(\mathrm{A})$ and is

defined as cl ${ }^{\mu}(A)=\cap\{B: B$ is a supra closed set and $A \subseteq B\}$.

(ii) The supra interior of a set $A$ is denoted by int ${ }^{\mu}(A)$ and defined as $\operatorname{int}^{\mu}(A)=\cup\{B: B$ is a supra open set and $A \supseteq B\}$.

Definition 2.3[8] Let $(X, \tau)$ be a topological spaces and $\mu$ be a supra topology on $X$. We call $\mu$ a supra topology associated with $\tau$ if $\tau \subset \mu$.

Definition 2.4[10] Let $(X, \mu)$ be a supra topological space. A set $A$ is called a supra b-open set if $A$ $\subseteq$ cl $^{\mu}\left(\right.$ int $\left.^{\mu}(A)\right) \cup$ int $^{\mu}\left({ }^{\mu} l^{\mu}(A)\right)$. The complement of a supra b-open set is called a supra b-closed set.

Definition 2.5[7] A subset A of a supra topological space $(X, \mu)$ is called $\mathrm{bT}^{\mu}{ }^{\mu}$-closed set if bcl ${ }^{\mu}(\mathrm{A}) \subseteq \mathrm{U}$ whenever $A \subseteq U$ and $U$ is $T^{\mu}$ - open in $(X, \mu)$.

Definition 2.6[9] Let $A$ and $B$ be subsets of $X$. Then $A$ and $B$ are said to be supra separated, if $\mathrm{A} \cap \mathrm{cl}^{\mathrm{\mu}}(\mathrm{B})=\mathrm{B} \cap \mathrm{cl}^{\mathrm{\mu}}(\mathrm{A})=\phi$.

Definition 2.7[5] Let $(X, \tau) \rightarrow(Y, \sigma)$ be two topological spaces and $\tau \subseteq \mu$. A function $f$ : $(\mathrm{X}, \tau) \rightarrow(\mathrm{Y}, \sigma)$ is called supra continuous, if the inverse image of each open set of $\mathrm{Y}$ is a supra open set in $\mathrm{X}$.

Definition 2.8[6] Let $(\mathrm{X}, \tau) \rightarrow(\mathrm{Y}, \sigma)$ be two topological spaces and $\mu$ and $\lambda$ be supra topologies associated with $\tau$ and $\sigma$ respectively. A function irresolute, if $\mathrm{f}^{-1}(\mathrm{~A})$ is supra open set of $\mathrm{X}$ for every supra open set $\mathrm{A}$ in $\mathrm{Y}$.

\section{Notations} closed set.

S-BTLC ${ }^{*}$ denotes supra $\mathrm{bT}^{*}$ - locally closed set and $\mathrm{S}^{\mathrm{BTLC}}{ }^{* *}$ denotes supra $\mathrm{bT}^{* *}-$ locally 
Bt-Locally Closed Sets And Bt-Locally Continuous Functions In Supra Topological Spaces.

\section{Definition 3.1}

\section{SUPRA bT-LOCALLY CLOSED SETS}

Let $(X, \mu)$ be a supra topological space. A subset $A$ of $(X, \mu)$ is called supra bT - locally closed set, if $\mathrm{A}=\mathrm{U} \cap \mathrm{V}$, where $\mathrm{U}$ is supra bT - open in $(\mathrm{X}, \mu)$ and $\mathrm{V}$ is supra bT - closed in $(\mathrm{X}, \mu)$. The collection of all supra bT- locally closed set $\mathrm{S}$ of $\mathrm{X}$ will be denoted by S-BTLC(X).

\section{Remark 3.2}

Every supra bT-closed set (resp. supra bT- open set) is S-BTLC.

\section{Definition 3.3}

For a subset $A$ of supra topological space $(X, \mu), A \in$ S-BTLC $^{*}(X, \mu)$, if there exist a supra bT- open set $\mathrm{U}$ and a supra closed set $\mathrm{V}$ of $(\mathrm{X}, \mu)$, respectively such that $\mathrm{A}=\mathrm{U} \cap \mathrm{V}$.

\section{Remark 3.4}

Every supra bT-closed set (resp. supra bT-open set) is S-BTLC*.

\section{Definition 3.5}

For a subset $A$ of supra topological space $(X, \mu), A \in \operatorname{S-BTLC}^{* *}(X, \mu)$, if there exist a supra open set $\mathrm{U}$ and a supra $\mathrm{bT}$ - closed set $\mathrm{V}$ of $(\mathrm{X}, \mu)$, respectively such that $\mathrm{A}=\mathrm{U} \cap \mathrm{V}$.

\section{Remark 3.6}

Every supra bT-closed set (resp. supra bT-open set) is S-BTLC ${ }^{* *}$.

Theorem 3.7 Let $A$ be a subset of $(X, \mu)$. If $A \in \operatorname{S-BTLC}^{*}(X, \mu)$ (or) $\operatorname{S-BTLC}^{* *}(X, \mu)$ then $A$ is S-BTLC $(\mathrm{X}, \mu)$.

Proof Given $A \in \mathrm{S}-B T L C^{*}(X, \mu)$ (or) $\mathrm{S}-B T L C^{* *}(X, \mu)$,by definition $A=U \cap V$, where U is supra bT- open set and V is supra closed set (or) U is supra open set and V is supra bT - closed set. By theorem 3.2 [7] every supra closed set is supra bT - closed set, therefore V is supra bT - closed set (or) every supra open set is supra bT - closed set, therefore U is supra bT- open set. Then A is S-BTLC $(X, \mu)$.

\section{Example 3.8}

Let $X=\{a, b, c\}$ and $\mu=\{X, \varphi,\{a\},\{b\},\{a, b\}\}$.In this $(X, \mu), S_{-B T L C}^{*}(X, \mu)$ and S-BTLC ${ }^{* *}(X, \mu)$ are the proper subset of S-BTLC $(X, \mu)$, because S-BTLC $(X, \mu)=P(X)$.

Theorem 3.9 Let $A$ be a subset of $(X, \mu)$. If $A \in S-L C(X, \mu)$ then $A$ is $\operatorname{S-BTLC}(X, \mu)$.

Proof Given $A \in S-L C$,by definition $A=U \cap V$, where $U$ is supra open set and $V$ is supra closed set. Since every supra open set is supra bT - open set and every supra closed set is supra bT - closed set. Then A $\in$ S$\operatorname{BTLC}(\mathrm{X}, \mu)$

\section{Example 3.10}

The converse of the above theorem is not true from the following example.

Theorem 3.11 For a subset $A$ of $(X, \mu)$, the following are equivalent:

(i) $\quad \mathrm{A} \in \mathrm{S}^{-\mathrm{BTLC}^{* *}}(\mathrm{X}, \mu)$

(ii) $\quad \mathrm{A}=\mathrm{U} \cap \mathrm{bcl}^{\mu}(\mathrm{A})$, for some supra open set $\mathrm{U}$

(iii) $\quad b c l^{\mu}(\mathrm{A})-\mathrm{A}$ is supra $\mathrm{bT}$ - closed

(iv) $\quad \mathrm{A} \cup\left[\mathrm{X}-\mathrm{bcl}{ }^{\mu}(\mathrm{A})\right]$ is supra $\mathrm{bT}-$ open

Proof (i) $\Rightarrow$ (ii): Given $A \in \operatorname{S-BTLC}^{* *}(X, \mu)$, then there exist a supra open subset $U$ and a supra bT- closed subset $V$ such that $A=U \cap V$. Since $A \subset U$ and $A \subset b c l^{\mu}(A)$, then $A \subset U \cap b c l^{\mu}(A)$.

Conversely, we have $b \mathrm{cl}^{\mu}(\mathrm{A}) \subset \mathrm{V}$ and hence $\mathrm{A}=\mathrm{U} \cap \mathrm{V} \supset \mathrm{U} \cap \mathrm{bcl}^{\mu}(\mathrm{A})$. Therefore $\mathrm{A}=\mathrm{U} \cap \mathrm{bcl}{ }^{\mu}(\mathrm{A})$.

(ii) $\Rightarrow(\mathrm{i})$ : Let $\mathrm{A}=\mathrm{U} \cap \mathrm{bcl}^{\mu}(\mathrm{A})$, for some supra open set $\mathrm{U}$. Clearly, bcl ${ }^{\mu}(\mathrm{A})$ is supra $\mathrm{bT}$ - closed and hence $\mathrm{A}=$

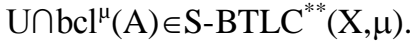

(ii) $\Rightarrow$ (iii): Let $A=U \cap b c l^{\mu}(A)$, for some supra open set $U$. Then $A \in \operatorname{S}_{-B T L C}{ }^{* *}(X, \mu)$. This implies $U$ is supra open and $b_{c l}^{\mu}(A)$ is supra $b T$ - closed. Therefore $b_{c l}^{\mu}(A)-A$ is supra bT- closed.

(iii) $\Rightarrow$ (ii): $\quad$ Let $U=X-\left[b c l^{\mu}(A)-A\right]$.By (iii) $U$ is supra bT- open in $X$. We know that every supra open is supra bT- open, therefore $U$ is supra open in $X$. Then $A=U \cap b c l^{\mu}(A)$ holds.

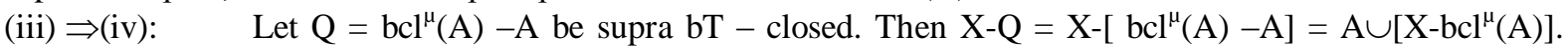
Since $\mathrm{X}-\mathrm{Q}$ is supra $\mathrm{bT}$ - open, $\mathrm{A} \cup\left[\mathrm{X}-\mathrm{bcl} \mathrm{l}^{\mu}(\mathrm{A})\right]$ is supra $\mathrm{bT}-$ open.

(iv) $\Rightarrow\left(\right.$ iii): $\quad$ Let $U=A \cup\left[X-b c l^{\mu}(A)\right]$. Since $X-U$ is supra $b T-\operatorname{closed}$ and $X-U=b c l^{\mu}(A)-A$ is supra $b T-$ closed.

\section{Definition 3.12}

Let $\mathrm{A}$ be subset of $(\mathrm{X}, \mu)$. Then

(i) The supra bT-closure of a set A is denoted by $b T-c l^{\mu}(A)$, define as $b T-c l^{\mu}(A)=\cap\{B: B$ is supra bTclosed and $\mathrm{A} \subseteq \mathrm{B}\}$. 
(ii) The supra bT-interior of a set A is denoted by $b T-$ int $^{\mu}(\mathrm{A})$, define as $b T-\operatorname{int}^{\mu}(\mathrm{A})=\cap\{\mathrm{B}: \mathrm{B}$ is supra $\mathrm{bT}$ open and $\mathrm{B} \subseteq \mathrm{A}\}$.

Theorem 3.13 For a subset $\mathrm{A}$ of $(X, \mu)$, the following are equivalent:

(i) $\quad \mathrm{A} \in \mathrm{S}-\mathrm{BTLC}(\mathrm{X}, \mu)$

(ii) $\quad \mathrm{A}=\mathrm{U} \cap \mathrm{bT}-\mathrm{cl}^{\mu}(\mathrm{A})$, for some supra $\mathrm{bT}$ - open set $\mathrm{U}$

(iii) $\quad \mathrm{bT}_{-\mathrm{cl}}^{\mu}(\mathrm{A})-\mathrm{A}$ is supra bT- closed

(iv) $\quad \mathrm{A} \cup\left[\mathrm{X}-\mathrm{bT}-\mathrm{cl}^{\mu}(\mathrm{A})\right]$ is supra $\mathrm{bT}$ - open

Proof $\quad(\mathrm{i}) \Rightarrow(\mathrm{ii}): \quad$ Given $\quad \mathrm{A} \in \mathrm{S}$-BTLC $(\mathrm{X}, \mu)$, then there exist a supra bT - open subset $\mathrm{U}$ and a supra bTclosed subset $V$ such that $A=U \cap V$. Since $A \subset U$ and $A \subset b T-c^{\mu}(A)$, then $A \subset U \cap b T-c l^{\mu}(A)$.

Conversely, we have $b T-c^{\mu}(A) \subset V$ and hence $A=U \cap V \supset U \cap b T-c^{\mu}(A)$. Therefore $A=U \cap b T-c l^{\mu}(A)$.

(ii) $\Rightarrow$ (i): Let $A=U \cap b T-c l^{\mu}(A)$, for some supra bT - open set U. Clearly, bT-cl ${ }^{\mu}(A)$ is supra bT- closed and hence $\mathrm{A}=\mathrm{U} \cap \mathrm{bT}-\mathrm{cl}^{\mu}(\mathrm{A}) \in \mathrm{S}-\mathrm{BTLC}(\mathrm{X}, \mu)$.

(ii) $\Rightarrow$ (iii): Let $A=U \cap b T-c^{\mu}(A)$, for some supra bT - open set $U$. Then $A \in \operatorname{S-BTLC}(X, \mu)$. This implies $U$ is supra bT - open and bT-cl ${ }^{\mu}(A)$ is supra $b T$ - closed. Therefore $b T-c l^{\mu}(A)-A$ is supra bT- closed.

(iii) $\Rightarrow$ (ii): Let $U=X-\left[b^{-}-l^{\mu}(A)-A\right]$.By (iii) $U$ is supra bT- open in X. Then $A=U \cap b T-c l^{\mu}(A)$ holds.

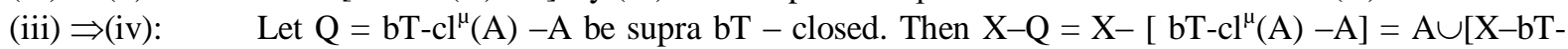
$\left.\mathrm{cl}^{\mu}(\mathrm{A})\right]$. Since $\mathrm{X}-\mathrm{Q}$ is supra $\mathrm{bT}-$ open, $\mathrm{A} \cup\left[\mathrm{X}-\mathrm{bT}-\mathrm{cl}^{\mu}(\mathrm{A})\right]$ is supra bT -open.

(iv) $\Rightarrow$ (iii): $\quad$ Let $U=A \cup\left[X-b T-c^{\mu}(A)\right]$. Since $X-U$ is supra $b T-\operatorname{closed}$ and $X-U=b T-l^{\mu}(A)-A$ is supra bT - closed.

Theorem 3.14 For a subset $\mathrm{A}$ of $(\mathrm{X}, \mu)$, the following are equivalent:

(i) $\quad \mathrm{A} \in \operatorname{S-BTLC}^{*}(\mathrm{X}, \mu)$

(ii) $\quad \mathrm{A}=\mathrm{U} \cap \mathrm{cl}^{\mu}(\mathrm{A})$, for some supra bT - open set $\mathrm{U}$

(iii) $\quad \mathrm{bcl}^{\mu}(\mathrm{A})-\mathrm{A}$ is supra $\mathrm{bT}$ - closed

(iv) $\quad \mathrm{A} \cup\left[\mathrm{X}-\mathrm{bcl}^{\mu}(\mathrm{A})\right]$ is supra $\mathrm{bT}$ - open

Proof $\quad(\mathrm{i}) \Rightarrow(\mathrm{ii})$ : Given $\mathrm{A} \in \mathrm{S} \mathrm{BTLC}^{*}(\mathrm{X}, \mu)$, then there exist a supra bT - open subset $\mathrm{U}$ and a supra closed subset $V$ such that $A=U \cap V$. Since $A \subset U$ and $A \subset c l^{\mu}(A)$, then $A \subset U \cap c l^{\mu}(A)$.

Conversely, we have $\mathrm{cl}^{\mu}(\mathrm{A}) \subset \mathrm{V}$ and hence $\mathrm{A}=\mathrm{U} \cap \mathrm{V} \supset \mathrm{U} \cap \mathrm{cl}{ }^{\mu}(\mathrm{A})$. Therefore $\mathrm{A}=\mathrm{U} \cap \mathrm{cl}^{\mu}(\mathrm{A})$.

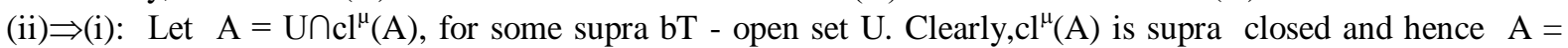
$\mathrm{U}_{\mathrm{cl}}{ }^{\mu}(\mathrm{A}) \in \mathrm{S}^{-\mathrm{BTLC}^{*}}(\mathrm{X}, \mu)$.

(ii) $\Rightarrow$ (iii): Let $A=U \cap c l^{\mu}(A)$, for some supra bT - open set $U$. Then $A \in \operatorname{S}_{\text {-BTLC }}^{*}(X, \mu)$. This implies $U$ is supra bT - open and $\mathrm{cl}^{\mu}(\mathrm{A})$ is supra closed. Therefore $\mathrm{cl}^{\mu}(\mathrm{A})-\mathrm{A}$ is supra closed. We know that every supra closed is supra bT- closed, therefore $\mathrm{bcl}^{\mu}(\mathrm{A})-\mathrm{A}$ is supra bT - closed.

(iii) $\Rightarrow$ (ii): Let $U=X-\left[b^{\mu} l^{\mu}(A)-A\right]$.By (iii) $U$ is supra bT- open in $X$. Then $A=U \cap c l^{\mu}(A)$ holds.

(iii) $\Rightarrow\left(\right.$ iv): $\quad$ Let $Q=b c l^{\mu}(A)-A$ be supra bT -closed. Then $X-Q=X-\left[b^{\mu} l^{\mu}(A)-A\right]=A \cup\left[X-b c l^{\mu}(A)\right]$. Since $\mathrm{X}-\mathrm{Q}$ is supra $\mathrm{bT}$ - open, $\mathrm{A} \cup\left[\mathrm{X}-\mathrm{bcl} \mathrm{l}^{\mu}(\mathrm{A})\right]$ is supra $\mathrm{bT}$ - open.

(iv) $\Rightarrow$ (iii): $\quad$ Let $U=A \cup\left[X-b c l^{\mu}(A)\right]$. Since $X-U$ is supra $b T$-closed and $X-U=b c l^{\mu}(A)-A$ is supra $b T$ closed.

Theorem 3.15 For a subset $A$ of $(X, \mu)$, if $A \in \operatorname{S-BTLC}^{* *}(X, \mu)$, then there exist an supra open set $G$ such that $\mathrm{A}=\mathrm{G} \cap \mathrm{bT}-\mathrm{cl}^{\mu}(\mathrm{A})$.

Proof Let $A \in S-B T L C C^{* *}(X, \mu)$. Then $A=G \cap V \Rightarrow A \subset G$. Then $A \subset b T-c l^{\mu}(A)$. Therefore, $A \subset G \cap b T-c^{\mu}(A)$. Also, we have $b T-c^{\mu}(A) \subset V$. This implies $A=G \cap V \supset G \cap b T-c^{\mu}(A) \Rightarrow A \supset G \cap b T-c^{\mu}(A)$. Thus $A=G \cap b T-c^{\mu}(A)$.

Theorem 3.16 For a subset $A$ of $(X, \mu)$, if $A \in \operatorname{S-BTLC}^{* *}(X, \mu)$, then there exist an supra open set $G$ such that $\mathrm{A}=\mathrm{G} \cap \mathrm{bcl}^{\mu}(\mathrm{A})$.

Proof Let $A \in S-B T L C C^{* *}(X, \mu)$. Then $A=G \cap V$, where $G$ is supra open set and V is supra bT-closed set. Then $\mathrm{A}=\mathrm{G} \cap \mathrm{V} \Rightarrow \mathrm{A} \subset \mathrm{G}$. Obviously, $\mathrm{A} \subset \mathrm{bcl}^{\mathrm{\mu}}(\mathrm{A})$.

Therefore, $A \subset G \cap b^{\mu}{ }^{\mu}(A)$. --------------(1)

Also, we have $b^{\mu}{ }^{\mu}(A) \subset V$. This implies $A=G \cap V \supset G \cap b c l^{\mu}(A) \Rightarrow A \supset G \cap b c l^{\mu}(A)$

From (1) and (2), we get $A=G \cap \operatorname{bcl}^{\mu}(A)$.

Theorem 3.17 Let $A$ be a subset of $(X, \mu)$. If $A \in \operatorname{S-BTLC}^{* *}(X, \mu)$, then $b T-c^{\mu}(A)-A$ is supra bT- closed and $\mathrm{A} \cup\left[\mathrm{X}-\mathrm{bT}-\mathrm{cl}^{\mu}(\mathrm{A})\right]$ is supra bT - open.

Proof Given $A \in \operatorname{S-BTLC}^{* *}(\mathrm{X}, \mu)$. Then there exist a supra open subset $\mathrm{U}$ and a supra bT- closed subset V such that $\mathrm{A}=\mathrm{U} \cap \mathrm{V}$. This implies $\mathrm{bT}-\mathrm{cl}^{\mu}(\mathrm{A})$ is supra $\mathrm{bT}$ - closed. Therefore, $\mathrm{bT}-\mathrm{cl}^{\mu}(\mathrm{A})-\mathrm{A}$ is supra bT- closed. Also, $\left[\mathrm{X}-\left[\mathrm{bT}-\mathrm{cl}^{\mu}(\mathrm{A})-\mathrm{A}\right]\right]=\mathrm{A} \cup\left[\mathrm{X}-\mathrm{bT}-\mathrm{cl}^{\mu}(\mathrm{A})\right]$. Therefore $\mathrm{A} \cup\left[\mathrm{X}-\mathrm{bT}-\mathrm{cl}^{\mu}(\mathrm{A})\right]$ is supra bT - open.

Remark 3.18 The converse of the above theorem need not be true as seen from the following example.

Example 3.19 Let $X=\{a, b, c, d\} \quad$ and $\mu \quad(X, \phi,\{a\},\{b\},\{a, b\}\}$.Then $\{X, \phi,\{a\},\{b\},\{c\},\{d\},\{a, c\},\{a, d\},\{b, c\},\{b, d\},\{c, d\},\{a, c, d\},\{b, c, d\}\}$ is the set of all supra bT-closed set in $X$ 
and $\mathrm{S}-\mathrm{BTLC} \mathrm{C}^{* *}(\mathrm{X}, \mu)=\mathrm{P}(\mathrm{X})-\{\{\mathrm{a}, \mathrm{b}, \mathrm{c}\},\{\mathrm{a}, \mathrm{b}, \mathrm{d}\}\}$.If $\mathrm{A}=\{\mathrm{a}, \mathrm{b}, \mathrm{c}\}$, then $\mathrm{bT}-\mathrm{cl}{ }^{\mu}(\mathrm{A})-\mathrm{A}=\{\mathrm{d}\}$ is supra $\mathrm{bT}-$ closed and $\mathrm{A} \cup\left[\mathrm{X}-\mathrm{bT}-\mathrm{cl}^{\mu}(\mathrm{A})\right]=\mathrm{A}$ is supra $\mathrm{bT}-$ open but $\mathrm{A} \notin \mathrm{S}-\mathrm{BTLC} \mathrm{C}^{* *}(\mathrm{X}, \mu)$.

Theorem 3.20 If $A \in \operatorname{S-BTLC}^{*}(X, \mu)$ and $B$ is supra open, then $A \cap B \in \operatorname{S-BTLC}^{*}(X, \mu)$.

Proof Suppose $A \in S_{-B T L C}(X, \mu)$, then there exist a supra bT- open set $U$ and supra closed set $V$ such that $\mathrm{A}=\mathrm{U} \cap \mathrm{V}$. So $\mathrm{A} \cap \mathrm{B}=\mathrm{U} \cap \mathrm{V} \cap \mathrm{B}=(\mathrm{U} \cap \mathrm{B}) \cap \mathrm{V}$, where $\mathrm{U} \cap \mathrm{B}$ is supra bT- open. Therefore, $\mathrm{A} \cap \mathrm{B} \in \mathrm{S}$ $\operatorname{BTLC}^{*}(\mathrm{X}, \mu)$.

Theorem 3.21 If $A \in \operatorname{S-BTLC}(X, \mu)$ and $B$ is supra open, then $A \cap B \in \operatorname{S-BTLC}(X, \mu)$.

Proof Suppose $A \in S-B T L C(X, \mu)$, then there exist a supra bT- open set $U$ and supra $b T$ - closed set $V$ such that $\mathrm{A}=\mathrm{U} \cap \mathrm{V}$. So $\mathrm{A} \cap \mathrm{B}=\mathrm{U} \cap \mathrm{V} \cap \mathrm{B}=(\mathrm{U} \cap \mathrm{B}) \cap \mathrm{V}$, where $\mathrm{U} \cap \mathrm{B}$ is supra bT- open. Therefore, $\mathrm{A} \cap \mathrm{B} \in \mathrm{S}$ $\operatorname{BTLC}(\mathrm{X}, \mu)$.

Theorem 3.22 If $A \in \operatorname{S-BTLC}^{* *}(X, \mu)$ and $B$ is supra closed, then $A \cap B \in \operatorname{S-BTLC}^{* *}(X, \mu)$.

Proof Suppose $A \in \operatorname{S-BTLC}^{*}(X, \mu)$, then there exist a supra open set $U$ and supra bT- closed set $\mathrm{V}$ such that $\mathrm{A}=\mathrm{U} \cap \mathrm{V}$. So $\mathrm{A} \cap \mathrm{B}=\mathrm{U} \cap \mathrm{V} \cap \mathrm{B}=\mathrm{U} \cap(\mathrm{B} \cap \mathrm{V})$, where $\mathrm{B} \cap \mathrm{V}$ is supra bT- closed. Hence, $\mathrm{A} \cap \mathrm{B} \in \mathrm{S}$ $\operatorname{BTLC}^{* *}(\mathrm{X}, \mu)$.

\section{SUPRA bT- LOCALLY CONTINUOUS FUNCTIONS}

In this section, we define a new type of functions called supra bT- locally continuous functions (SBTL -continuous functions),supra bT - locally irresolute functions(S-BTL-irresolute) and study some of their properties.

\section{Definition 4.1}

Let $(X, \tau)$ and $(Y, \sigma)$ be two topological spaces and $\tau \subseteq \mu$. A function $\mathrm{f}:(\mathrm{X}, \tau) \rightarrow(\mathrm{Y}, \sigma)$ is called S-BTLcontinuous (resp., S-BTL"- continuous, and S-BTL ${ }^{* *}$ - continuous), if $f^{-1}(\mathrm{~A}) \in \mathrm{S}-\mathrm{BTLC}(\mathrm{X}, \mu),\left(\right.$ resp., $f^{-1}$ (A) $\in \operatorname{S-BTLC}^{*}(\mathrm{X}, \mu)$, and $\left.f^{-1}(\mathrm{~A}) \in \mathrm{S}_{-\mathrm{BTLC}^{* * *}}(\mathrm{X}, \mu)\right)$ for each $\mathrm{A} \in \sigma$.

\section{Definition 4.2}

Let $(\mathrm{X}, \tau)$ and $(\mathrm{Y}, \sigma)$ be two topological spaces and $\mu$ and $\lambda$ be a supra topologies associated with $\tau$ and $\sigma$ respectively.A function $\mathrm{f:}(\mathrm{X}, \tau) \rightarrow(\mathrm{Y}, \sigma)$ is said to be S-BTL-irresolute(resp., S-BTL ${ }^{*}$ - irresolute, resp., SBTL $^{* *}$-irresolute) if $f^{-1}$ (A) $\in \mathrm{S}$-BTLC $(\mathrm{X}, \mu),\left(\right.$ resp., $f^{-1}(\mathrm{~A}) \in \mathrm{S}-\mathrm{BTLC}{ }^{*}(\mathrm{X}, \mu)$, resp., $f^{-1}$ (A) $\in \mathrm{S}$ $\left.\operatorname{BTLC}^{* *}(X, \mu)\right)$ for each $A \in \operatorname{S-BTLC}(Y, \lambda)\left(\right.$ resp., $A \in S-B T L C(Y, \lambda)$, resp., $\left.A \in \operatorname{S-BTLC}^{* *}(Y, \lambda)\right)$.

Theorem 4.3 Let $(\mathrm{X}, \tau)$ and $(\mathrm{Y}, \sigma)$ be two topological spaces and $\mu$ be a supra topology associated with $\tau$. Let $\mathrm{f}:(\mathrm{X}, \tau) \rightarrow(\mathrm{Y}, \sigma)$ be a function. If $\mathrm{f}$ is $\mathrm{S}-\mathrm{BTL}^{*}$ - continuous (or) S-BTL**- continuous, then it is S-BTLcontinuous.

Proof Let $\mathrm{f}:(\mathrm{X}, \tau) \rightarrow(\mathrm{Y}, \sigma)$ be a function. If $\mathrm{f}$ is S-BTL ${ }^{*}$ - continuous (or) S-BTL**- continuous, by definition $f^{-1}(\mathrm{~A}) \in \mathrm{S}-\mathrm{BTLC}^{*}(\mathrm{X}, \mu)$, and $f^{-1}(\mathrm{~A}) \in \mathrm{S}^{-\mathrm{BTLC}^{* *}}(\mathrm{X}, \mu)$ for each $\mathrm{A} \in \sigma$. By theorem $3.7, f^{-1}(\mathrm{~A}) \in \mathrm{S}$ $\operatorname{BTLC}(X, \mu)$. Then it is S-BTL- continuous.

Theorem 4.4 Let $(\mathrm{X}, \tau)$ and $(\mathrm{Y}, \sigma)$ be two topological spaces and $\mu$ and $\lambda$ be a supra topologies associated with $\tau$ and $\sigma$ respectively. Let $\mathrm{f}:(\mathrm{X}, \tau) \rightarrow(\mathrm{Y}, \sigma)$ be a function . If $\mathrm{f}$ is S-BTL-irresolute(resp., S-BTL ${ }^{*}$ - irresolute, and S-BTL ${ }^{* *}$-irresolute), then it is S-BTL- continuous( resp., S-BTL - continuous, and S-BTL ${ }^{* *}$ - continuous).

Proof Let $f:(X, \tau) \rightarrow(Y, \sigma)$ be a function . Let A is supra closed of $Y$. By theorem 3.2[7] every supra closed set is supra bT-closed set, therefore A is supra bT - closed set. Since f is S-BTL-irresolute(resp., S-BTL ${ }^{*}$ irresolute, and S-BTL ${ }^{* *}$-irresolute), $f^{-1}$ (A) is S-BTL-closed. Therefore $\mathrm{f}$ is S-BTL-continuous ( resp., S-BTL ${ }^{*}$ continuous, and S-BTL ${ }^{* *}$ - continuous).

Theorem 4.5 If $\mathrm{g}: \mathrm{X} \rightarrow \mathrm{Y}$ is $\mathrm{S}-\mathrm{BTL}-$ continuous and $\mathrm{h}: \mathrm{Y} \rightarrow \mathrm{Z}$ is supra continuous, then hog: $\mathrm{X} \rightarrow \mathrm{Z}$ is S-BTLcontinuous.

Proof Let $\mathrm{g}: \mathrm{X} \rightarrow \mathrm{Y}$ is $\mathrm{S}$-BTL- continuous and $\mathrm{h}: \mathrm{Y} \rightarrow \mathrm{Z}$ is supra continuous. By definition, $\mathrm{g}^{-1}(\mathrm{~V}) \in \mathrm{S}$ $\operatorname{BTLC}(X), V \in Y$ and $h^{-1}(W) \in Y, W \in Z$. Let $W \in Z$, then $(h o g)^{-1}(W)=\left(g^{-1} h^{-1}\right)(W)=g^{-1}\left(h^{-1}(W)\right)=g^{-1}(V)$, for $\mathrm{V} \in \mathrm{Y}$. This implies, $(\mathrm{hog})^{-1}(\mathrm{~W})=\mathrm{g}^{-1}(\mathrm{~V}) \in \mathrm{S}-\mathrm{BTLC}(\mathrm{X}), \mathrm{W} \in \mathrm{Z}$. Hence hog is S-BTL- continuous.

Theorem 4.6 If $\mathrm{g}: \mathrm{X} \rightarrow \mathrm{Y}$ is S-BTL-irresolute and $\mathrm{h}: \mathrm{Y} \rightarrow \mathrm{Z}$ is S-BTL-continuous, then $\mathrm{hog}: \mathrm{X} \rightarrow \mathrm{Z}$ is $\mathrm{S}$ BTL- continuous.

Proof Let $\mathrm{g}: \mathrm{X} \rightarrow \mathrm{Y}$ is S-BTL-irresolute and $\mathrm{h}: \mathrm{Y} \rightarrow \mathrm{Z}$ is S-BTL-continuous. By definition, $\quad \mathrm{g}^{-1}(\mathrm{~V}) \in \mathrm{S}$ $\operatorname{BTLC}(X)$, for $V \in S-B T L C(Y)$ and $h^{-1}(W) \in S-B T L C(Y)$, for $W \in Z$. Let $W \in Z$, then $(h o g)^{-1}(W)=\left(g^{-1} h^{-1}\right)(W)=g^{-}$ ${ }^{1}\left(h^{-1}(W)\right)=g^{-1}(V)$, for $V \in S-B T L C(Y)$. This implies, $(h o g)^{-1}(W)=g^{-1}(V) \in S-B T L C(X), W \in Z$. Hence hog is SBTL- continuous.

Theorem 4.7 If $\mathrm{g}: \mathrm{X} \rightarrow \mathrm{Y}$ and $\mathrm{h}: \mathrm{Y} \rightarrow \mathrm{Z}$ are S-BTL- irresolute, then hog $: \mathrm{X} \rightarrow \mathrm{Z}$ is S-BTL- irresolute. 
Proof By the hypothesis and the definition, we have $\mathrm{g}^{-1}(\mathrm{~V}) \in \mathrm{S}-\mathrm{BTLC}(\mathrm{X})$, for $\mathrm{V} \in \mathrm{S}-\mathrm{BTLC}(\mathrm{Y})$ and and $h^{-1}(W) \in S-B T L C(Y)$, for $W \in S-B T L C(Z)$. Let $W \in S-B T L C(Z)$, then $(h o g)^{-1}(W)=\left(g^{-1} h^{-1}\right)(W)=g^{-1}\left(h^{-}\right.$ $\left.{ }^{1}(\mathrm{~W})\right)=\mathrm{g}^{-1}(\mathrm{~V})$, for $\mathrm{V} \in \mathrm{S}-\mathrm{BTLC}(\mathrm{Y})$. Therefore, $(h \circ)^{-1}(\mathrm{~W})=\mathrm{g}^{-1}(\mathrm{~V}) \in \mathrm{S}-\mathrm{BTLC}(\mathrm{X}), \mathrm{W} \in \mathrm{S}-\mathrm{BTLC}(\mathrm{Z})$. Thus, hog is S-BTL- irresolute.

\section{References}

[1] D.Andrijevic”,On b- open sets", mat.Vesnik 48(1996), no.1-2,59-64.

[2] Arokiarani .I, Balachandran. K and Ganster .M, " Regular Generalized locallyclosed sets and RGL-continuous functions", Indian J.pure appl. Math., 28 (5): May(1997) 661-669.

[3] Bharathi. S, Bhuvaneswari.K,and Chandramathi.N, "Generalization of locally b- closed sets",International Journal of Applied Engineering Research,Volume 2,(2011) No 2.

[4] Bourbaki, "General Topology", Part I, Addison - Wesley(Reading,Mass) (1996).

[5] Devi.R., Sampathkumar S. \& Caldas M.," On supra $\alpha$-open sets and S $\alpha$ - continuous functions", General Mathematics, 16(2),(2008),77-84.

[6] Ganster M.\& Reilly I.L.," Locally closed sets and LC continuous functions", International J.Math. and Math.Sci.,12,(1989)417424.

[7] Krishnaveni.K and Vigneshwaran.M, "On bT-Closed sets in supra topological Spaces, On supra bT- closed sets in supra topological spaces", International journal of Mathematical Archive-4(2),2013,1-6.

[8] A.S.Mashhour, A.A.Allam, F.S.Mohamoud and F.H.Khedr,"On supra topological spaces", Indian J.Pure and Appl.Math.No.4, 14 (1983),502-510.

[9] Ravi.O., Ramkumar. G \& Kamaraj .M ,'On supra g-closed sets”, International Journal of Advances in pure and Applied Mathematics, 1(2), [2010] 52-66.

[10] O.R. Sayed and Takashi Noiri," On b-open sets and supra b-Continuity on opological spaces", European Journal of Pure and applied Mathematics, 3(2)(2010), 295-302. 